\title{
COMPORTAMENTO DA DISTRIBUIÇÃO DO SUPORTE DE PESO EM PÉ EM PESSOAS COM HEMIPARESIA CRÔNICA: REVISÃO COM METANÁLISE
}

\author{
STANDING WEIGHT-BEARING DISTRIBUTION BEHAVIOR IN PEOPLE WITH CHRONIC \\ HEMIPARESIS: REVIEW AND META-ANALYSIS

\section{Monike Barros Camargos ${ }^{a}$, Hudson Azevedo Pinheiro ${ }^{b}$, Michelle Rabeloc, Roberto de Souza Baptistad, Emerson Fachin-Martins ${ }^{\mathrm{e}}$}

amonikecamargos@gmail.com, bhudsonap@gmail.com, cmichellineribeiro@yahoo.com.br, dbaptista@ieee.org, eefmartins@unb.br Universidade de Brasília - Brasília (DF), Brasil

Data de recebimento do artigo: 02/03/2015

Data de aceite do artigo: 19/11/2015

\section{RESUMO}

Embora seja relatada a ideia de que pessoas com hemiparesia crônica possuem distribuição assimétrica do suporte de peso em pé, algumas evidências também apontam distribuiçóes simétricas nessa população. Esta revisão teve por objetivo levantar pesquisas que investigaram a distribuição do suporte de peso em pé em sujeitos que adquiriram hemiparesia crônica decorrente de acidente vascular encefálico (AVE) identificando qual comportamento de suporte de peso foi descrito pelos autores para essa populaçáo. Foram consultadas as bases de dados PubMed, EMBASE, Cochrane, CINAHL e SciELO, até outubro de 2015. Das referências selecionadas foram destacadas as informaçóes: (1) medidas de distribuição do suporte de peso; (2) equipamentos utilizados para medir essa distribuição; (3) informaçôes disponíveis sobre as propriedades psicométricas do instrumento utilizado e (4) ideia apresentada sobre o tipo de distribuiçấo no suporte de peso. Dentre as referências encontradas, aquelas que apresentaram dados obtidos a partir de balanças digitais e que permitiam o cálculo de uma razão de simetria foram utilizadas para metanálise. Observou-se na maioria dos estudos, assim como mencionado previamente, que prevaleceu a ideia de que os indivíduos com hemiparesia crônica possuem distribuição assimétrica do suporte de peso com sobrecarga do hemicorpo náo parético. No entanto, a heterogeneidade dos estudos, a falta de parâmetros para se definir quais são os limites do que seria simetria no suporte de peso e as poucas informaçōes sobre as propriedades psicométricas dos instrumentos utilizados apontam para necessidade de estudos adicionais.

Palavras-chave: Transtornos cerebrovasculares; hemiplegia; equilíbrio postural; suporte de carga; revisão.

\section{ABSTRACT}

Although it has been reported that people with chronic hemiparesis present asymmetric distribution of weight in the standing position, some evidences also show symmetric distribution in this population. This review aimed to collect researches investigating weight-bearing distribution in the standing position on patients with hemiparesis after stroke, identifying which weight-bearing behavior has been described by authors for this population. PUBMED, EMBASE, COCHRANE, CINAHL and SCIELO databases were consulted until October 2015. It was highlighted from the selected references the following information: (1) measurements of weight-bearing distribution, (2) devices used to measure this distribution, (3) available information about psychometric properties of the device used and (4) presented concept of weight-bearing distribution. Among the references collected, those presenting data obtained by digital scales and allowing calculation of symmetry ratio were used for meta-analysis. In most of studies, as previous mentioned, prevailed the concept that subjects with chronic hemiparesis have asymmetric distribution overloading non-paretic hemibody. However, the heterogeneity among studies, the lack of parameters to define weight-bearing symmetry and little information about psychometric properties of the devices used show the necessity of further investigation.

Keywords: Cerebrovascular disorders; hemiplegia; postural balance; weight-bearing; review. 


\section{Introdução}

Instabilidades posturais estão comumente presentes em sobreviventes de acidente vascular encefálico (AVE) e sáo descritas na hemiparesia crônica como estando associadas às deficiências estruturais e funcionais que promovem uma distribuição assimétrica no suporte de peso entre os hemicorpos ${ }^{1-4}$.

Os profissionais que trabalham com a reabilitação física costumam aceitar a ideia de que as pessoas que adquiriram hemiparesia apresentam, na posiçâo ortostática, distribuição assimétrica do suporte de peso com sobrecarga sobre o hemicorpo não parético ${ }^{2-5}$.

Fundamentados nessa ideia, muitos profissionais estabelecem programas de reabilitação cujos objetivos incluem a induçáo da simetria nesta postura, justificada no pressuposto de que a simetria esteja associada a ganhos funcionais.

Entretanto, ao observar evidências científicas que estudaram o suporte de peso de pessoas com hemiparesia, pouco consenso se encontra quanto aos reais benefícios de um suporte simétrico nesta postura, sendo encontrados tanto resultados que apontam ganhos funcionais em indivíduos com suporte mais simétrico ${ }^{1,2,6}$ quanto resultados que sugerem ser o desempenho funcional náo afetado pela assimetria ${ }^{7,8}$. Além disso, mais recentemente foi descrito que pessoas com hemiparesia podem apresentar diferentes comportamentos de suporte de peso em pé, inclusive suporte simétrico, desde que seja estabelecida uma referência dos limites que definem o que é uma distribuição simétrica ${ }^{5,9,10}$.

Considerando as divergências encontradas, antes de se estabelecer a ideia de que a simetria esteja associada ou não a ganhos funcionais é necessário melhor definir o comportamento de suporte de peso em pé desses indivíduos. Assim, esta revisão de literatura teve como objetivo sistematizar informaçóes de estudos que avaliaram o comportamento de suporte de peso em pé em sujeitos com hemiparesia crônica decorrente de AVE, estruturando uma metanálise dos parâmetros que permitiram isso.

\section{Metodologia}

\section{Estratégias de busca}

Artigos publicados até outubro de 2015 foram levantados nas bases de dados PubMed, EMBASE, Cochrane, CINAHL e SciELO, utilizando combinação e sinônimos dos descritores: "acidente vascular encefálico", "postura", "equilíbrio", "distribuição de peso", "suporte de carga", "bípede", "postura ereta" e "funcional".

Foram incluídas nesta revisão as referências que: (1) possuíam amostras compostas por adultos capazes de permanecerem de pé; (2) trabalharam com sujeitos com hemiparesia adquirida a um mínimo de seis meses após AVE e (3) utilizaram medidas de distribuiçẫo do suporte de peso na posição de pé. Para as referências que apresentavam estudos com intervençáo terapêutica, foram consideradas somente as medidas obtidas antes da intervenção. Foram excluídas da revisão as referências que: (1) não especificavam faixa etária da sua amostra; (2) utilizavam indivíduos com hemiparesia adquirida por outras causas que não fossem cerebrovasculares e (3) investigavam sujeitos na fase aguda/subaguda após o AVE (menos de seis meses após o evento).

Dois examinadores analisaram os resumos de cada uma das referências levantadas para identificar os critérios de elegibilidade. Uma vez incluída, era localizada na íntegra, e suas referências bibliográficas foram checadas para identificar possíveis referências não encontradas nas buscas iniciais, mas que pudessem atender aos critérios de inclusão. Um terceiro examinador solucionou divergências.

\section{Extração e análise das informações}

As informaçóes dos artigos selecionados foram extraídas por dois examinadores, sendo que o terceiro examinador foi consultado no caso de divergências. Foram extraídos os seguintes dados dos artigos selecionados: a) autores e ano de publicaçáa; b) qualidade do estudo; c) delineamento do estudo; d) número de participantes; e) presença ou náo de grupo controle; f) medida de simetria/ assimetria na distribuição do suporte de peso; g) cálculo da medida de simetria/assimetria no suporte de peso; h) instrumento utilizado para avaliação da distribuição do peso; i) avaliação ou não das propriedades psicométricas do instrumento utilizado; j) ideia apresentada pelos autores sobre distribuição do suporte de peso (Tabela 1).

A qualidade das referências foi determinada pelo índice Newcastle-Ottawa $(\mathrm{NOS})^{11}$, para os estudos transversais de caso controle, e pela escala PEDro de Fisioterapia em Evidência ${ }^{12}$, para os ensaios clínicos randomizados. Os estudos que não possuíam grupo controle não puderam ter sua qualidade metodológica analisada. Dois examinadores avaliaram a qualidade dos estudos selecionados.

A razão de simetria (RS) foi extraída dos estudos que utilizaram balanças digitais para medida da distribuição do suporte de peso, sendo definida pela divisão dos valores do peso aferidos no hemicorpo parético sobre os valores do hemicorpo não parético conforme descrito previamente em outros estudos $5,9,10$.

Utilizou-se o software Comprehensive MetaAnalysis 3.0 (Biostat, Englewood, NJ) e adotou-se intervalo de confiança de $95 \%$. O gráfico de forest-plot foi construído com o software Excel considerando a média e o desvio-padrão consultados nas referências. 
Tabela 1: Informações identificadas para as referências bibliográficas selecionadas e sistematizadas nesta revisão.

\begin{tabular}{|c|c|c|c|c|c|c|c|c|c|c|}
\hline \# & Referência & $\begin{array}{l}\text { Quali- } \\
\text { dade }\end{array}$ & $\begin{array}{l}\text { Delinea- } \\
\text { mento }\end{array}$ & $\begin{array}{c}\text { Amos- } \\
\text { tra }\end{array}$ & $\begin{array}{l}\text { Grupo } \\
\text { con- } \\
\text { trole? }\end{array}$ & $\begin{array}{l}\text { Medida de } \\
\text { simetria }\end{array}$ & $\begin{array}{c}\text { Como foi calculada } \\
\text { a medida? }\end{array}$ & $\begin{array}{l}\text { Instru- } \\
\text { mento }\end{array}$ & $\begin{array}{l}\text { Psico- } \\
\text { metria? }\end{array}$ & Ideia \\
\hline 1 & $\begin{array}{l}\text { Peurala et } \\
\text { al., } 2007\end{array}$ & $8^{*}$ & Transversal & $\mathrm{n}=45$ & Sim & Não especifica & $\begin{array}{l}\text { Somente descreve va- } \\
\text { lores de deslocamento } \\
\text { do centro de pressáo. }\end{array}$ & $\begin{array}{l}\text { Plataforma } \\
\text { de força }\end{array}$ & Não & $\begin{array}{l}\text { Assimetria com } \\
\text { sobrecarga do } \\
\text { hemicorpo náo } \\
\text { parético. }\end{array}$ \\
\hline 2 & $\begin{array}{l}\text { Hung et } \\
\text { al., } 2014\end{array}$ & $8^{* *}$ & $\begin{array}{l}\text { Ensaio } \\
\text { clínico } \\
\text { randomizado }\end{array}$ & $\mathrm{n}=30$ & Sim & $\begin{array}{l}\text { Medida relati- } \\
\text { va }(\%)\end{array}$ & $\begin{array}{l}\text { Porcentagem de peso } \\
\text { registrada no membro } \\
\text { parético. }\end{array}$ & $\begin{array}{l}\text { Plataforma } \\
\text { de força }\end{array}$ & Não & $\begin{array}{l}\text { Assimetria com } \\
\text { sobrecarga do } \\
\text { hemicorpo não } \\
\text { parético. }\end{array}$ \\
\hline 3 & $\begin{array}{l}\text { Martins et } \\
\text { al., } 2011\end{array}$ & $7^{*}$ & Transversal & $\mathrm{n}=20$ & Sim & $\begin{array}{l}\text { Razão de sime- } \\
\text { tria entre pés } \\
\text { (adimensional) }\end{array}$ & $\begin{array}{l}\text { Quociente do suporte } \\
\text { registrado em forma } \\
\text { de peso na balança em } \\
\text { cada pé. }\end{array}$ & $\begin{array}{c}\text { Balança } \\
\text { digital }\end{array}$ & Sim $* * *$ & $\begin{array}{l}\text { Simetria e } \\
\text { assimetria com } \\
\text { sobrecarga } \\
\text { tanto no hemi- } \\
\text { corpo parético } \\
\text { como no não } \\
\text { parético. }\end{array}$ \\
\hline 4 & $\begin{array}{l}\text { Lisinski et } \\
\text { al., } 2012\end{array}$ & $6^{* *}$ & $\begin{array}{l}\text { Ensaio } \\
\text { clínico } \\
\text { randomizado }\end{array}$ & $\mathrm{n}=26$ & Sim & $\begin{array}{l}\text { Medida relati- } \\
\text { va }(\%)\end{array}$ & $\begin{array}{l}\text { Porcentagem do } \\
\text { centro de pressáo em } \\
\text { cada pé. }\end{array}$ & $\begin{array}{l}\text { Plataforma } \\
\text { de força }\end{array}$ & Não & $\begin{array}{l}\text { Assimetria com } \\
\text { sobrecarga do } \\
\text { hemicorpo não } \\
\text { parético. }\end{array}$ \\
\hline 5 & $\begin{array}{l}\text { Dickstein, } \\
1984\end{array}$ & $6^{*}$ & Transversal & $\mathrm{n}=23$ & Sim & $\begin{array}{l}\text { Razão de } \\
\text { ortostatis- } \\
\text { mo lateral } \\
\text { entre os pés } \\
\text { (adimensional) }\end{array}$ & $\begin{array}{l}\text { Valor da área de } \\
\text { diâmetro circular } \\
\text { resultante da pressão } \\
\text { em função da carga } \\
\text { em cada pé. }\end{array}$ & $\begin{array}{l}\text { Baropodo- } \\
\text { metria } \\
\text { óptica }\end{array}$ & Não & $\begin{array}{l}\text { Assimetria com } \\
\text { sobrecarga do } \\
\text { hemicorpo náo } \\
\text { parético. }\end{array}$ \\
\hline 6 & $\begin{array}{l}\text { Lee et al., } \\
2015\end{array}$ & $5^{* *}$ & $\begin{array}{l}\text { Ensaio } \\
\text { clínico } \\
\text { randomizado }\end{array}$ & $\mathrm{n}=36$ & Sim & $\begin{array}{c}\text { Razão de } \\
\text { simetria } \\
\text { entre os pés } \\
\text { (adimensional) }\end{array}$ & $\begin{array}{l}\text { Quociente do suporte } \\
\text { registrado em cada pé. }\end{array}$ & $\begin{array}{l}\text { Baropodô- } \\
\text { metro }\end{array}$ & Não & $\begin{array}{l}\text { Assimetria com } \\
\text { sobrecarga do } \\
\text { hemicorpo náo } \\
\text { parético }\end{array}$ \\
\hline 7 & $\begin{array}{l}\text { Park e } \\
\text { Kang, } \\
2013\end{array}$ & $5^{* *}$ & $\begin{array}{l}\text { Ensaio } \\
\text { clínico } \\
\text { randomizado }\end{array}$ & $\mathrm{n}=20$ & Sim & $\begin{array}{l}\text { Medida relati- } \\
\text { va }(\%)\end{array}$ & $\begin{array}{l}\text { Porcentagem do com- } \\
\text { ponente da força de } \\
\text { reação com o solo. }\end{array}$ & $\begin{array}{l}\text { Plataforma } \\
\text { de força }\end{array}$ & Não & $\begin{array}{l}\text { Assimetria com } \\
\text { sobrecarga do } \\
\text { hemicorpo náo } \\
\text { parético. }\end{array}$ \\
\hline 8 & $\begin{array}{l}\text { Marigold e } \\
\text { Eng, } 2006\end{array}$ & $5^{*}$ & Transversal & $n=28$ & Sim & $\begin{array}{c}\text { Índice de } \\
\text { assimetria } \\
\text { (adimensional) }\end{array}$ & $\begin{array}{l}\text { Resultante da relação } \\
\text { entre a componente } \\
\text { vertical da força de } \\
\text { reação com o solo em } \\
\text { cada pé. }\end{array}$ & $\begin{array}{l}\text { Plataforma } \\
\text { de força }\end{array}$ & Sim & $\begin{array}{l}\text { Assimetria com } \\
\text { sobrecarga do } \\
\text { hemicorpo não } \\
\text { parético. }\end{array}$ \\
\hline 9 & $\begin{array}{l}\text { Itotani et } \\
\text { al., } 2015\end{array}$ & $5^{*}$ & Transversal & $\mathrm{n}=17$ & Sim & $\begin{array}{l}\text { Medida relati- } \\
\text { va }(\%)\end{array}$ & $\begin{array}{l}\text { Porcentagem do peso } \\
\text { registrada no membro } \\
\text { parético, em funçáo } \\
\text { do peso corporal. }\end{array}$ & $\begin{array}{l}\text { Dispositivo } \\
\text { com } \\
\text { sensores de } \\
\text { pressão }\end{array}$ & Não & $\begin{array}{l}\text { Assimetria com } \\
\text { sobrecarga do } \\
\text { hemicorpo não } \\
\text { parético. }\end{array}$ \\
\hline 10 & $\begin{array}{l}\text { Chen e } \\
\text { Wing, } \\
2012\end{array}$ & $5^{*}$ & Transversal & $\mathrm{n}=18$ & Sim & $\begin{array}{l}\text { Índice de as- } \\
\text { simetria entre } \\
\text { os pés }\end{array}$ & $\begin{array}{l}\text { Diferença do compo- } \\
\text { nente vertical da força } \\
\text { de reaçáo ao solo en- } \\
\text { tre os pés, em função } \\
\text { do peso corporal. }\end{array}$ & $\begin{array}{l}\text { Plataforma } \\
\text { de força }\end{array}$ & Não & $\begin{array}{l}\text { Assimetria com } \\
\text { sobrecarga do } \\
\text { hemicorpo não } \\
\text { parético. }\end{array}$ \\
\hline 11 & $\begin{array}{l}\text { Song e } \\
\text { Hwangbo, } \\
2015\end{array}$ & $4^{* *}$ & $\begin{array}{l}\text { Ensaio } \\
\text { clínico } \\
\text { randomizado }\end{array}$ & $\mathrm{n}=40$ & Sim & $\begin{array}{l}\text { Medida relati- } \\
\text { va }(\%)\end{array}$ & $\begin{array}{l}\text { Porcentagem do peso } \\
\text { corporal registrada no } \\
\text { membro parético. }\end{array}$ & $\begin{array}{l}\text { Sistema de } \\
\text { Análise com } \\
\text { Biofeedback }\end{array}$ & Não & $\begin{array}{l}\text { Assimetria com } \\
\text { sobrecarga do } \\
\text { hemicorpo não } \\
\text { parético. }\end{array}$ \\
\hline
\end{tabular}

continua... 
Tabela 1: Continuação.

\begin{tabular}{|c|c|c|c|c|c|c|c|c|c|c|}
\hline$\#$ & Referência & $\begin{array}{l}\text { Quali- } \\
\text { dade }\end{array}$ & $\begin{array}{l}\text { Delinea- } \\
\text { mento }\end{array}$ & $\begin{array}{c}\text { Amos- } \\
\text { tra }\end{array}$ & $\begin{array}{l}\text { Grupo } \\
\text { con- } \\
\text { trole? }\end{array}$ & $\begin{array}{l}\text { Medida de } \\
\text { simetria }\end{array}$ & $\begin{array}{c}\text { Como foi calculada } \\
\text { a medida? }\end{array}$ & $\begin{array}{l}\text { Instru- } \\
\text { mento }\end{array}$ & $\begin{array}{l}\text { Psico- } \\
\text { metria? }\end{array}$ & Ideia \\
\hline 12 & $\begin{array}{l}\text { Mansfield } \\
\text { et al., } 2013\end{array}$ & NA & Transversal & $\mathrm{n}=147$ & Não & $\begin{array}{c}\text { Media relativa } \\
(\%)\end{array}$ & $\begin{array}{l}\text { Porcentagem do com- } \\
\text { ponente da força de } \\
\text { reação com o solo. }\end{array}$ & $\begin{array}{l}\text { Plataforma } \\
\text { de } \\
\text { força }\end{array}$ & Não & $\begin{array}{c}\text { Simetrias e } \\
\text { assimetrias } \\
\text { com sobrecarga } \\
\text { tanto no hemi- } \\
\text { corpo parético } \\
\text { como no não } \\
\text { parético. }\end{array}$ \\
\hline 13 & $\begin{array}{l}\text { Adegoke et } \\
\text { al., } 2012\end{array}$ & NA & Transversal & $n=53$ & Não & $\begin{array}{l}\text { Medida relati- } \\
\text { va }(\%)\end{array}$ & $\begin{array}{l}\text { Porcentagem do peso } \\
\text { registrado em cada pé, } \\
\text { em função do peso } \\
\text { corporal. }\end{array}$ & $\begin{array}{l}\text { Balança } \\
\text { digital }\end{array}$ & Não & $\begin{array}{l}\text { Assimetria com } \\
\text { sobrecarga do } \\
\text { hemicorpo não } \\
\text { parético. }\end{array}$ \\
\hline 14 & $\begin{array}{l}\text { Lewek et } \\
\text { al., } 2014\end{array}$ & NA & Transversal & $\mathrm{n}=39$ & Não & $\begin{array}{l}\text { Medida relati- } \\
\text { va }(\%)\end{array}$ & $\begin{array}{l}\text { Porcentagem do com- } \\
\text { ponente da força de } \\
\text { reação com o solo. }\end{array}$ & $\begin{array}{l}\text { Plataforma } \\
\text { de força }\end{array}$ & Não & $\begin{array}{l}\text { Assimetria com } \\
\text { sobrecarga do } \\
\text { hemicorpo não } \\
\text { parético. }\end{array}$ \\
\hline 15 & $\begin{array}{l}\text { Don Kim } \\
\text { et al., } 2015\end{array}$ & NA & Transversal & $n=16$ & Não & $\begin{array}{c}\text { Valores de } \\
\text { carga }(\mathrm{N}) \text {, } \\
\text { normalizados } \\
\text { ou não pelo } \\
\text { peso corporal } \\
(\%)\end{array}$ & $\begin{array}{l}\text { Carga no membro } \\
\text { parético, normalizada } \\
\text { ou não pelo peso } \\
\text { corporal. }\end{array}$ & $\begin{array}{l}\text { Plataforma } \\
\text { de força }\end{array}$ & Não & $\begin{array}{l}\text { Assimetria com } \\
\text { sobrecarga do } \\
\text { hemicorpo não } \\
\text { parético. }\end{array}$ \\
\hline 16 & $\begin{array}{l}\text { Eng e Chu, } \\
2002\end{array}$ & NA & Transversal & $\mathrm{n}=15$ & Não & $\begin{array}{l}\text { Medida relati- } \\
\text { va }(\%)\end{array}$ & $\begin{array}{l}\text { Porcentagem da com- } \\
\text { ponente vertical da } \\
\text { força de reação com o } \\
\text { solo em cada pé. }\end{array}$ & $\begin{array}{l}\text { Plataforma } \\
\text { de força }\end{array}$ & Sim & $\begin{array}{l}\text { Assimetria com } \\
\text { sobrecarga do } \\
\text { hemicorpo não } \\
\text { parético. }\end{array}$ \\
\hline 17 & $\begin{array}{l}\text { Pereira et } \\
\text { al., } 2010\end{array}$ & NA & Transversal & $\mathrm{n}=14$ & Não & $\begin{array}{l}\text { Razão de sime- } \\
\text { tria entre pés } \\
\text { (adimensional) }\end{array}$ & $\begin{array}{l}\text { Quociente do suporte } \\
\text { registrado em forma } \\
\text { de peso na balança em } \\
\text { cada pé. }\end{array}$ & $\begin{array}{c}\text { Balança } \\
\text { digital }\end{array}$ & Não & $\begin{array}{c}\text { Simetria e } \\
\text { assimetria com } \\
\text { sobrecarga } \\
\text { tanto no hemi- } \\
\text { corpo parético } \\
\text { como no não } \\
\text { parético. }\end{array}$ \\
\hline 18 & $\begin{array}{l}\text { Aruin et } \\
\text { al., } 2000\end{array}$ & NA & $\begin{array}{c}\text { Quasi- } \\
\text { experimental }\end{array}$ & $\mathrm{n}=8$ & Não & $\begin{array}{l}\text { Medida relati- } \\
\text { va }(\%)\end{array}$ & $\begin{array}{l}\text { Porcentagem da com- } \\
\text { ponente vertical da } \\
\text { força de reaçáo com o } \\
\text { solo em cada pé. }\end{array}$ & $\begin{array}{l}\text { Plataforma } \\
\text { de força }\end{array}$ & Não & $\begin{array}{l}\text { Assimetria com } \\
\text { sobrecarga do } \\
\text { hemicorpo não } \\
\text { parético. }\end{array}$ \\
\hline 19 & $\begin{array}{l}\text { Balthazar } \\
\text { et al., } 2012\end{array}$ & NA & Transversal & $\mathrm{n}=5$ & Não & $\begin{array}{l}\text { Razão de sime- } \\
\text { tria entre pés } \\
\text { (adimensional) }\end{array}$ & $\begin{array}{l}\text { Quociente do suporte } \\
\text { registrado em forma } \\
\text { de peso na balança em } \\
\text { cada pé. }\end{array}$ & $\begin{array}{l}\text { Balança } \\
\text { digital }\end{array}$ & Não & $\begin{array}{c}\text { Simetria e } \\
\text { assimetria com } \\
\text { sobrecarga } \\
\text { tanto no hemi- } \\
\text { corpo parético } \\
\text { como no não } \\
\text { parético. }\end{array}$ \\
\hline 20 & $\begin{array}{l}\text { Marklund } \\
\text { e Klassbo, } \\
2006\end{array}$ & NA & $\begin{array}{l}\text { Experimental } \\
\text { com série de } \\
\text { casos }\end{array}$ & $\mathrm{n}=5$ & Não & $\begin{array}{l}\text { Valores de } \\
\text { carga em cada } \\
\text { pé }(\mathrm{kg})\end{array}$ & $\begin{array}{l}\text { Foi utilizado o valor } \\
\text { indicado no visor. }\end{array}$ & $\begin{array}{c}\text { Balança } \\
\text { digital }\end{array}$ & Não & $\begin{array}{l}\text { Assimetria com } \\
\text { sobrecarga do } \\
\text { hemicorpo não } \\
\text { parético. }\end{array}$ \\
\hline
\end{tabular}

Referências organizadas por ordem de qualidade, sendo que aquelas indicadas com * tiveram a qualidade avaliada pelo índice de Newcastle-Ottawa Scale e aquela indicada com ${ }^{* *}$ teve a qualidade avaliada pela escala PEDro. As referências cuja qualidade não foi avaliada foram identificadas na coluna pela sigla NA - Não Avaliados, nestes casos a ordem de qualidade foi data pelo tamanho da amostra. Na referência 3, a marcação *** indica que apesar de ter apresentado informaçôes de propriedades psicométricas, foi para população de sujeitos sem hemiparesia. 


\section{Resultados}

A busca inicial resultou em 87 resumos. Após análise dos critérios de inclusão, considerou-se 67 resumos relevantes, dos quais resultou em 20 selecionados para a revisão final (Figura 1).

Das vinte referências, onze foram avaliados quanto à qualidade (Tabela 1). Dentre as referências com qualidade avaliada, seis estudos náo apresentaram boa qualidade metodológica, sendo três transversais ${ }^{13-15}$ e três ensaios clínicos randomizados ${ }^{16-18}$. A Tabela 1 permitiu observar que a maioria dos artigos utilizou delineamento observacional transversal (65\%), sendo encontrados ainda cinco ensaios clínicos randomizados (25\%) e uma minoria de estudos de caráter mais descritivo (10\%).

O tamanho das amostras variou muito de um estudo para outro e somente $55 \%$ das pesquisas utilizaram grupo controle, sendo que somente os estudos com grupo controle puderam ter sua qualidade metodológica avaliada. As formas de se medir simetria no suporte também variou entre os estudos. Em 55\% dos estudos utilizaram como instrumento plataformas de força, $25 \%$ usaram balanças digitais, e dois estudos utilizaram sistema de baropodometria.

Somente $15 \%$ das referências descreveram ou calcularam propriedades psicométricas das medidas, sendo que, em uma delas, as propriedades psicométricas foram extraídas de medidas feitas em pessoas sem hemiparesia.

$\mathrm{Na}$ maioria dos estudos, as medidas de simetria/ assimetria na distribuição do suporte de peso foram apresentadas em funçáo do peso corporal, na forma de porcentagem do peso ${ }^{19,14,18,20,21}$ ou do componente vertical da força de reação do solo no membro inferior parético ou não parético ${ }^{17,22,23-25}$. Em contrapartida, estudos remanescentes calcularam índice ou razão de simetria entre os membros afetado e não afetado ${ }^{6}$, além de deslocamento do centro de pressão ${ }^{26}$, área do diâmetro circular resultante da pressão, em função da carga em cada pé $^{27}$, ou apresentaram somente valores brutos referentes a carga nos membros inferiores ${ }^{28}$.

A maioria das pesquisas $(80 \%)$ consideraram que o sujeito com hemiparesia possui um comportamento de suporte de peso assimétrico com sobrecarga do hemicorpo nâo parético, diante de $20 \%$ de estudos que apontaram evidências de que nessa populaçáo é possível observar outros comportamentos como simetria e assimetria com sobrecarga do hemicorpo parético.

A metanálise feita com as quatro referências $5,29,30,28$ que permitiram um cálculo de razão de simetria (RS) e apontaram uma média abaixo de 1 que indica uma sobrecarga no hemicorpo não parético (Figura 2). Entretanto, o desvio-padráo de alguns estudos e da própria resultante da metanálise apontaram a possibilidade de RS indicativa de comportamento simétrico e assimétrico com sobrecarga do hemicorpo parético. Um dos estudos que utilizou balança digital na avaliação da distribuição do suporte de peso foi excluído da metanálise, porque a RS média dos participantes estava fora do intervalo de confiança de $95 \%{ }^{20}$.

Figura 1: Fluxograma de busca e seleção das referências utilizadas no estudo.

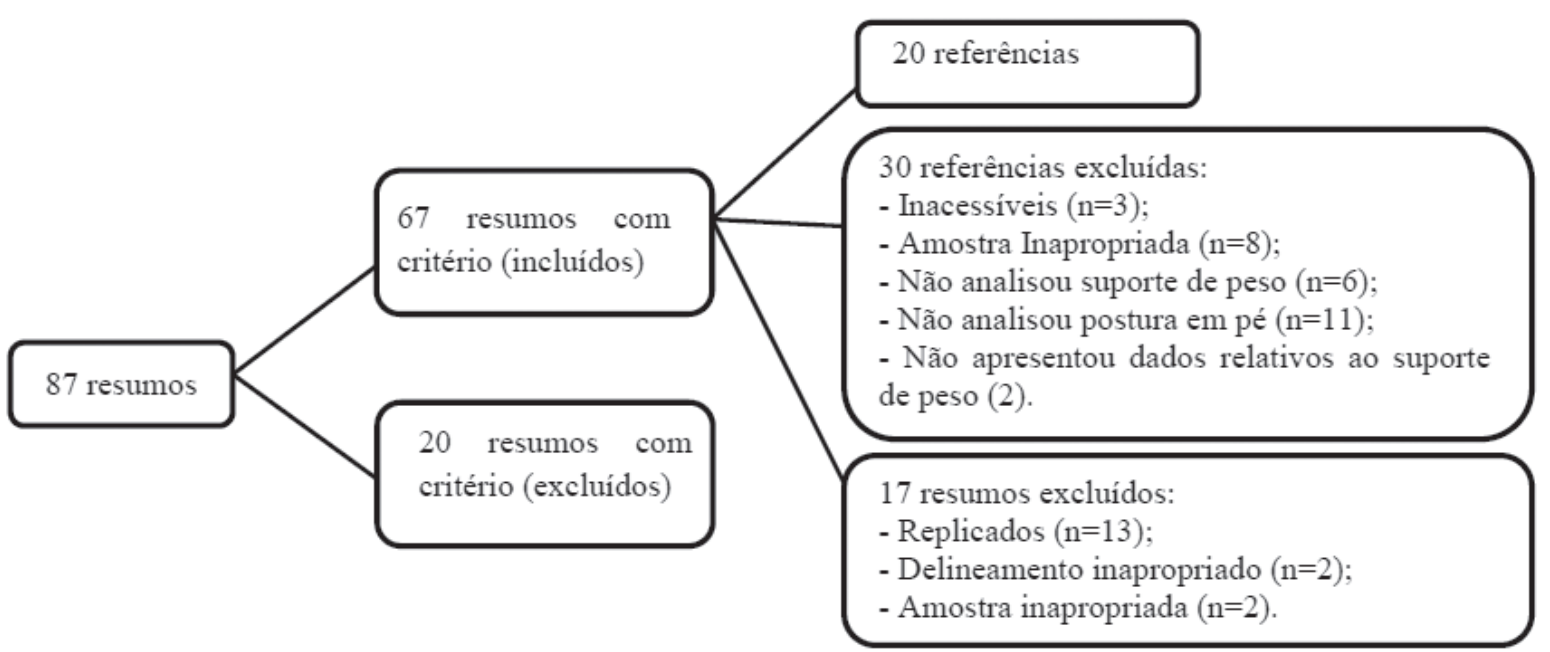


Figura 2: Forest-plot da metanálise das referências que permitiram calcular razão de simetria. Losangos negros indicam a média e a linha contínua, o desvio-padrão das razões de simetria de cada referência. O losango maior branco indica a média e desvio-padrão de todas as referências. A linha vertical descontínua indica razão de simetria igual a 1 (suporte simétrico).

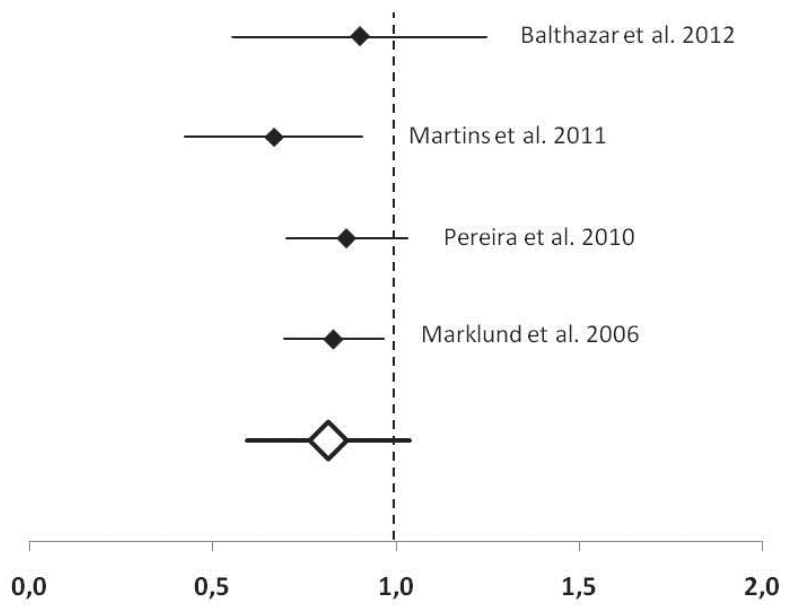

\section{Discussão}

Esta revisão mostrou que informaçóes sobre o comportamento de distribuição do suporte na postura em pé são bastante descritas na população de pessoas com hemiparesia (67 resumos relevantes foram encontrados). Ainda, apontou que esse comportamento é estudado por meio de uma variedade de medidas adquirida em três principais instrumentos: as plataformas de força; as plataformas de baropodometria e as balanças digitais. As plataformas de força são consideradas, pela maioria dos trabalhos, o padrấo ouro para esse tipo de análise $\mathrm{e}^{10,31-35}$.

Apesar da diversidade de publicaçôes na temática de distribuição do suporte de peso na postura em pé de sujeitos com hemiparesia, quando a busca dessa temática é combinada por meio dos termos suporte de carga ou suporte de peso (Weight-bearing, na versão em inglês), um número limitado de referências foi encontrado (87 resumos). A análise mais detalhada das referências na íntegra mostrou uma variedade de métodos publicados para tratar de um mesmo objeto. Diversidade maior ainda foi observada quanto às medidas obtidas por esses métodos, o que possibilitou uma metanálise de apenas quatro estudos (Figura 2).

O tamanho da amostra nos estudos variou consideravelmente, sendo encontrado desde amostras bem pequenas em trabalhos que apresentaram estudos de caso ou série de casos ${ }^{29,31}$ até amostras maiores com mais de 10 sujeitos. Estudo analisando recrutamento, presença e adesáo a protocolos de tratamento por sobreviventes de
AVE mostrou que a cada 150 pacientes triados em 14 meses para protocolo de ensaio clínico randomizado, 10 foram recrutados, tendo sido observadas taxa de presença de $72 \%$ e adesão de $97 \%$ ao protocolo ${ }^{37}$. Tais dificuldades metodológicas poderiam justificar o tamanho reduzido das amostras observado em algumas referências.

Nesta revisão, os estudos que não utilizaram controles para definir parâmetros de simetria tenderam a adotar a tradicional ideia de que o suporte de peso de pessoas com hemiparesia crônica é caracterizado pela distribuição assimétrica com sobrecarga no hemicorpo não-parético. Essa ideia também é predominante na literatura científica que trata do assunto ${ }^{13-28,37}$.

Entretanto, evidências mais recentes que usaram padróes de referência para definir assimetria no suporte inseriram achados que incluem novas perspectivas de análise do suporte de peso na condição de hemiparesia, apresentando sujeitos com hemiparesia em comportamentos distintos do tradicionalmente aceito, ou seja, sujeitos cujo suporte de peso em pé se caracteriza por simetria na distribuição $0^{9,10,22,29,30,38}$ e assimetria com sobrecarga no hemicorpo parético ${ }^{39,40}$.

A metanálise dos dados sugere que simetria e assimetria para o lado náo parético poderiam ser mascaradas pelo comportamento predominante e tradicionalmente já aceito (assimetria com sobrecarga do hemicorpo náo parético). Uma vez adotados métodos que evidenciassem os demais comportamentos de suporte de peso em pé, novas consideraçóes e interpretaçôes poderiam ser refletidas sobre a real contribuição da simetria em proporcionais vantagens ou desvantagens funcionais para essa população.

Segundo alguns dos trabalhos analisados nesta revisão $0^{2,6}$, a assimetria no suporte de peso poderia representar uma estratégia adaptativa para compensar os déficits motores e somatossensoriais do membro afetado, permitindo ao indivíduo gerar os ajustes posturais necessários para manutençáo da estabilidade postural em uma situaçáo de assimetria. Essa nova perspectiva de análise sugere que as pessoas com hemiparesia poderiam ser treinadas em programas de reabilitação com diferentes estratégias a depender do comportamento de suporte utilizado para garantir sua estabilidade postu-

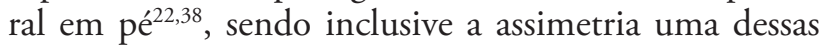
estratégias ${ }^{29}$.

Pioneiramente introduzido em 2011 por Martins, Araujo Barbosa, Menezes, Sousa e Costa e já utilizado como referência por outros autores ${ }^{22,38}$, a inclusão de um grupo controle que permitiu definir os limites de simetria contribuiu para análises mais detalhadas quanto aos tipos de suporte de peso e sua influência nas aquisições funcionais em programas de reabilitação. Observou-se nesta revisão sistemática que a ausência de critérios para julgar o que é assimetria no suporte 
promoveu divergências nos resultados dos estudos selecionados, indicando que tal cuidado metodológico deve ser aplicado.

\section{Conclusão}

Este estudo confirmou uma predominância da tradicional ideia de que as pessoas que convivem com sua deficiência hemiparética a mais de seis meses (crônica) possuem assimetria na distribuição do suporte de peso em pé com sobrecarga no hemicorpo não parético. Entretanto, ao considerar a heterogeneidade dos estudos, a falta de parâmetros de quais são os limites de simetria e as poucas informaçôes sobre as propriedades psicométricas dos instrumentos utilizados; é possível concluir que estudos mais padronizados, bem parametrizados quanto ao comportamento assimétrico e adequadamente instrumentalizados poderiam introduzir novas perspectivas a serem consideradas na definição de indução de simetria nos programas de tratamento de reabilitação da condição de hemiparesia.

\section{Referências}

1. Cheng PT, Liaw MY, Wong MK, Tang FT, Lee MY, Lin PS. The sit-to-stand movement in stroke patients and its correlation with falling. Arch Phys Med Rehabil. 1998;79(9):1043-6.

2. Genthon N, Rougier P, Gissot AS, Froger J, Pelissier J, Perennou D. Contribution of each lower limb to upright standing in stroke patients. Stroke. 2008;39(6):1793-9.

3. Geurts AC, Haart M, Nes IJ van, Duysens J. A review of standing balance recovery from stroke. Gait Posture. 2005;22(3):267-81.

4. Tyson SF, Hanley M, Chillala J, Selley A, Tallis RC. Balance disability after stroke. Phys Ther. 2006;86(1):30-8.

5. Martins EF, Araujo Barbosa PH, Menezes LT, Sousa PH, Costa AS. Is it correct to always consider weight-bearing asymmetrically distributed in individuals with hemiparesis? Physiother Theory Pract. 2011;27(8):566-71.

6. Chagas EF, Tavares MCGCF. A simetria e transferência de peso do hemiplégico: relação dessa condição com o desempenho de suas atividades funcionais. Fisioter. Pesqui. 2001;8(1):40-50.

7. Camargos AC, Rodrigues-de-Paula-Goulart F, TeixeiraSalmela LF. The effects of foot position on the performance of the sit-to-stand movement with chronic stroke subjects. Arch Phys Med Rehabil. 2009;90(2):314-9.

8. Teixeira-Salmela LF, Lima RCM, Lima LAO, Morais SG, Goulart F. Assimetria e desempenho funcional em hemiplégicos crônicos antes e após programa de treinamento em academia. Rev Bras Fisioter. 2005;9(2):227-33.
9. Martins EF, Barbosa PHFdeA, Menezes LT, Sousa PHC, Costa AS. Comparação entre medidas de descarga, simetria e transferência de peso em indivíduos com e sem hemiparesia. Fisioter Pesqui. 2011;18(3):228-34.

10. Menezes LTd, Barbosa PHFdA, Costa AS, Mundim AC, Ramos GC, Paz CCdSC, et al. Baropodometric technology used to analyze types of weight-bearing during hemiparetic upright position. Fisioter. Movi. 2012; 25(3):583-94.

11. Wells GA, Shea B, O'Connell D, Peterson JVW, Losos M, et al. The Newcastle-Ottawa Scale (NOS) for assessing the quality of nonrandomised studies in meta-analyses. [acesso em 2013 Sept. 1]; Available from: http://www.ohri.ca/programs/clinical_epidemiology/nosgen.pdf.

12. Maher CG, Sherrington C, Herbert RD, Moseley AM, Elkins M. Reliability of the PEDro scale for rating quality of randomized controlled trials. Phys Ther. 2003;83(8):713-21.

13. Marigold DS, Eng JJ. The relationship of asymmetric weight-bearing with postural sway and visual reliance in stroke. Gait Posture. 2006;23(2):249-55.

14. Itotani K, Murakami M, Itotani M, Nagai A, Imabori Y, Fujimoto K, Tanaka M, Kato J. Relationship between the weight-bearing ratio on the affected lower extremity and gait ability using a portable electronic foot sensor shoe $\left(\right.$ Step $\left.\mathrm{Aid}^{\circledR}\right)$ in hemiplegic stroke patients. J. Phys. Ther. Sci. 2015;27(2):321-3.

15. Chen HY, Wing AM. Independent control of force and timing symmetry in dynamic standing balance: Implications for rehabilitation of hemiparetic stroke patients. Hum Mov Sci. 2012;31(6):1660-9.

16. Lee H, Kim H, Ahn M, You Y. Effects of proprioception training with exercise imagery on balance ability of stroke patients. J Phys Ther Sci. 2015 Jan;27(1):1-4.

17. Park CS, Kang KY. The effects of additional action observational training for functional electrical stimulation treatment on weight bearing, stability and gait velocity of hemiplegic patients. J. Phys. Ther. Sci. 2013;25(3):1173-5.

18. Song G, Hwangbo G. The effect of a rehabilitational sliding machine and conventional neurological physical therapy on the balance of patients with hemiplegia. J. Phys. Ther. Sci. 2015;27(1):171-3.

19. Hung JW, Chou CX, Hsieh YW, Wu WC, Yu MY, Chen PC, Chang HF, Ding SE. Randomized comparison trial of balance training by using exergaming and conventional weight-shift therapy in patients with chronic stroke. Arch Phys Med Rehabil. 2014;95(9):1629-37.

20. Adegoke BO, Olaniyi O, Akosile CO. Weight bearing asymmetry and functional ambulation performance in stroke survivors. Glob J Health Sci. 2012;4(2):87-94.

21. Don Kim K, Lee HJ, Lee MH, Hwangbo G. Effect of ankle-foot orthosis on weight bearing of chronic stroke patients performing various functional standing tasks. J Phys. Ther. Sci. 2015;27(4):1059-61.

22. Mansfield A, Danells CJ, Zettel JL, Black SE, McIlroy WE. Determinants and consequences for standing 
balance of spontaneous weight-bearing on the paretic side among individuals with chronic stroke. Gait Posture. 2013;38(3):428-32.

23. Lewek MD, Bradley CE, Wutzke CJ, Zinder SM. The relationship between spatiotemporal gait asymmetry and balance in individuals with chronic stroke. J Appl Biomech. 2014;30(1):31-6.

24. Eng JJ, Chu KS. Reliability and comparison of weight-bearing ability during standing tasks for individuals with chronic stroke. Arch Phys Med Rehabil. 2002;83(8):1138-44.

25. Aruin AS, Hanke T, Chaudhuri G, Harvey R, Rao N. Compelled weightbearing in persons with hemiparesis following stroke: the effect of a lift insert and goal-directed balance exercise. J Rehabil Res Dev. 2000;37(1):65-72.

26. Peurala SH, Könönen P, Pitkänen K, Sivenius J, Tarkka IM. Postural instability in patients with chronic stroke. Restor Neurol Neurosci. 2007;25(2):101-8.

27. Lisinski P, Huber J, Gajewska E, Szlapinski P. The body balance training effect on improvement of motor functions in paretic extremities in patients after stroke. A randomized, single blinded trial. Clin Neurol Neurosurg. 2012;114(1):31-6.

28. Marklund I, Klassbo M. Effects of lower limb intensive mass practice in poststroke patients: single-subject experimental design with long-term follow-up. Clin Rehabil. 2006;20(7):568-76.

29. Pereira LC, Botelho AC, Martins EF. Relationships between body symmetry during weight-bearing and functional reach among chronic hemiparetic patients. Rev Bras Fisioter. 2010;14(3):229-66.

30. Balthazar RB, de Sousa PHC, de Araujo Barbosa PHF, de Menezes LT, Costa AS, Carneiro DVA, da Silva Maráes VRF, Martins EF. Could heart rate variability be associated with weight-bearing asymmetries in cerebrovascular diseases? International Journal of Case Reports and Images 2012;3(2):1-5.

31. Hurkmans HL, Bussmann JB, Benda E, Verhaar JA, Stam HJ. Techniques for measuring weight bearing during standing and walking. Clin Biomech (Bristol, Avon). 2003;18(7):576-89.

32. Bohannon RW, Tinti-Wald D. Accuracy of weightbearing estimation by stroke versus healthy subjects. Percept Mot Skills. 1991;72(3 Pt 1):935-41.

33. Bohannon RW, Waters G, Cooper J. Perception of unilateral lower extremity weightbearing during bilateral upright stance. Percept Mot Skills. 1989;69(3 Pt 1):875-80.

34. Rothstein JM. Measurement in Physical Therapy. New York: Churchill Livingstone; 1985.

35. Winstein CJ, Pohl PS, Cardinale C, Green A, Scholtz L, Waters CS. Learning a partial-weight-bearing skill: effectiveness of two forms of feedback. Phys Ther. 1996;76(9):985-93.

36. Scianni A, Teixeira-Salmela LF, Ada L. Challenges in recruitment, attendance and adherence of acute stroke survivors to a randomized trial in Brazil: a feasibility study. Rev Bras Fisioter. 2012;16(1):40-5.

37. Dickstein R, Nissan M, Pillar T, Scheer D. Foot-ground pressure pattern of standing hemiplegic patients. Major characteristics and patterns of improvement. Phys Ther. 1984;64(1):19-23.

38. Lu RR, Li F, Wu Y, Hu YS, Xu XL, Zou RL, Hu XF. Demonstration of posturographic parameters of squat-stand activity in hemiparetic patients on a new multi-utility balance assessing and training system. J Neuroeng Rehabil. 2013;10:37. doi: 10.1186/1743-0003-10-37.

39. Babyar SR, Peterson MG, Bohannon R, Perennou D, Reding M. Clinical examination tools for lateropulsion or pusher syndrome following stroke: a systematic review of the literature. Clin Rehabil. 2009;23(7):639-50.

40. Johannsen L, Broetz D, Karnath HO. Leg orientation as a clinical sign for pusher syndrome. BMC Neurol. 2006;6:30. doi: 10.1186/1471-2377-6-30.

\section{Como citar este artigo:}

Camargos MB, Pinheiro HÁ, Rabelo M, Baptista RS, Fachin-Martins E. Comportamento da distribuição do suporte de peso em pé em pessoas com hemiparesia crônica: revisão com metanálise. Rev. Aten. Saúde. 2016;14(48):86-93. 